\title{
Alimentação afetiva no cuidado em saúde mental: um relato de experiência
}

\author{
Welington de Lima Borges ${ }^{1}$ \\ Helicínia Giordana Espíndola Peixoto \\ Laryssa Fernandes de Souza Coelho ${ }^{3}$
}

\footnotetext{
${ }^{1}$ Nutricionista Residente do Programa de Saúde Mental do Adulto da Fundação de Ensino e Pesquisa em Ciências da Saúde (FEPECS)

2 Nutricionista Vice-coordenadora da Comissão de Residência Multiprofissional de Saúde e em Área Profissional da Saúde - COREMU da Fundação de Ensino e Pesquisa em Ciências da Saúde (FEPECS)

${ }^{3}$ Nutricionista Preceptora do Programa de Atenção Cardíaca da Fundação de Ensino e Pesquisa em Ciências da Saúde (FEPECS)
}

\section{RESUMO}

Introdução: A alimentação é necessária à vida, mas não se resume ao seu papel biológico de fornecer nutrientes para a manutenção da vitalidade. No âmbito da saúde mental, possibilita autonomia, buscando entender a singularidade da relação do usuário com a comida. Objetivo: Este trabalho objetiva relatar a experiência de um nutricionista residente do Programa de Residência Multiprofissional de Saúde Mental do Adulto em relação a importância da alimentação afetiva como potencializador da manutenção e/ou melhora da saúde mental. Métodos: Trata-se de um estudo de abordagem qualitativa, descritivo, do tipo relato de experiência, sobre o cuidado nutricional entre agosto de 2020 a fevereiro de 2021, baseado nas preferências alimentares de duas usuárias em internação psiquiátrica hospitalar. Resultados: Proporcionar autonomia nas escolhas alimentares, dentro das preferências do paciente, é possível por meio de estratégias como a gastronomia hospitalar, que por meio de adaptações contribui para a melhor aceitação, além de valorizar as características sensoriais da comida e seus simbolismos e valor cultural. Conclusão: Respeitar as escolhas do usuário é possível e contribui para a reabilitação psicossocial no cuidado em saúde mental.

Palavras-chave: Saúde mental, alimentação afetiva, comida

\section{Affective feeding in mental health care: an experience report}

\begin{abstract}
Introduction: Food is necessary for life, but it is not limited to its biological role of providing nutrients to maintain vitality. In the context of mental health, enabling autonomy, seeking to understand the uniqueness of each individual and their relationship with food. Objective:
\end{abstract}


This paper aims to report the experience of a resident nutritionist in the Multiprofessional Adult Mental Health Residency Program, and the importance of affective nutrition as an enhancer in the maintenance and/or improvement of mental health. Methods: This is a qualitative study, with descriptive approach, including experience report about nutritional care between August 2020 and February 2021. The study is based on the food preferences of two participants in a psychiatric hospitalization. Results: Providing autonomy in food choices within the participants' preferences is possible with strategies such as hospital gastronomy. Using adaptations in gastronomy contributes to better acceptance of ingredients, in addition to valuing the sensory characteristics of food, its symbolism, as well as cultural value. Conclusion: Respecting the patient's choices is possible and contributes to psychosocial rehabilitation in mental health care.

Keywords: Mental health, affective nutrition, food

\section{INTRODUÇÃO}

A alimentação é necessária para a manutenção da vida, sendo um determinante da vitalidade de um indivíduo, garantindo e fornecendo nutrientes necessários para o funcionamento e manutenção do corpo, além de favorecer a sensação de saciedade e bemestar, de modo que o ato de se alimentar é um comportamento instintivo inerente à natureza do ser humano, mas não se limita à função biológica ${ }^{1}$.

Embora sejam cotidianamente confundidos, há diferença semântica entre os termos "alimento" e "comida", de modo que alimento está ligado a função biológica, sendo tudo aquilo que possa fornecer nutrientes e contribuir para a manutenção da vida por meio da ingestão e digestão, entretanto, ser alimento não é determinante de ser comida, tendo em vista que comida é algo capaz de suprir além do biológico, não sendo apenas uma substância capaz de alimentar, mas um modo de ser e de comer, um estilo, um definidor de um grupo e um reforçador de identidade ${ }^{1}$.

Desde quando o ser humano descobriu o fogo, mudou a forma de como o alimento é preparado e consumido, e com isso trouxe mais significados ao ato de comer pois proporcionou o aumento das relações familiares em torno do alimento, fortalecendo assim os vínculos e agregando sentidos e experiencias coletivas, surgindo assim a comensalidade. $\mathrm{O}$ momento da alimentação possibilita não apenas eliminar a fome, mas favorece o ato de 
partilhar, entender processos, compartilhar e vivenciar experiências e sentimentos, sendo um importante potencializador de fortalecimento de vínculo entre os comensais ${ }^{2}$.

A contar do nosso nascimento temos a necessidade de nos alimentar, sendo geralmente o seio materno um dos principais meios para a garantia da alimentação, e também um dos primeiros contatos que possuímos com a comida. A amamentação é também um momento de trocas e de construção de relações com o outro e com a comida pois requer contato físico, atenção, cuidado e também proteção, dessa maneira a amamentação tende a ser um dos primeiros momentos capazes de agregar afeto à alimentação ${ }^{3}$.

Comer é também um momento de liberdade, de modo que se é possível escolher onde se come, como se come e o quê se come, há respeito à singularidade e diversidade entre indivíduos e coletivos. As escolhas e preferências alimentares são determinadas por inúmeros fatores externos: como a própria disponibilidade e cultura; e internos: como os sentimentos, afetos e memórias ${ }^{4}$.

O Brasil por meio de sua Política de Saúde Mental, iniciada desde a década de 1980, se tornou notável internacionalmente e amplamente estudado. Desde o princípio, a construção da Política de Saúde Mental esbarrou em dificuldades, seja pela demora como na consolidação da Lei de Saúde Mental, seja pela efetivação do cuidado baseado na liberdade e no território, rompendo com a lógica do cuidado hospitalocêntrico ${ }^{5}$.

De acordo com Amarante ${ }^{6}$ contar dos primórdios de sua história, o hospital exerceu um papel de exclusão, passando de local de caridade a controle social, e, finalmente um local medicalizador, focado na doença. O hospital psiquiátrico, embora comumente denominado de ambiente terapêutico, historicamente possui o papel de controle dos corpos com postura disciplinadora, geradora de perda de identidade e subjetividade, e facilitadora de fortalecimento de estigmas, retirando do "louco" sua voz e autonomia, sendo assim um local de mortificação do eu. 
Quando a doença é colocada em segundo plano e o indivíduo é evidenciado, pode-se então dar voz e ouvir, compreendendo suas necessidades, respeitando seu direito e espaço dentro da sociedade. Fornecer autonomia de escolhas dentro das limitações de cada um, e entender a perspectiva biopsicossocial, torna-se indispensável no cuidado em saúde mental, tornando assim as relações mais horizontais entre profissional e paciente, além de fortalecer o cuidado compartilhado, capaz de manter e recuperar a saúde, além de respeitar a singularidade de cada indivíduo ${ }^{6}$.

Este trabalho objetiva relatar a experiência de um nutricionista residente do Programa de Residência Multiprofissional de Saúde Mental do Adulto, e a importância da alimentação afetiva como potencializador da manutenção e/ou melhora da saúde mental.

\section{METODOLOGIA}

Trata-se de um estudo de abordagem qualitativa, descritivo, do tipo relato de experiência. De acordo com Minayo ${ }^{7}$, na pesquisa qualitativa há a preocupação com dados que não podem ser quantificados, mas que possui seu centro na compreensão e explicação de relações sociais, trabalhando assim com significados, aspirações, sentimentos, motivos, crenças e valores que permeiam as relações sociais e não podem ser reduzidos a nível de variáveis. Quanto à pesquisa descritiva, possui, como principal finalidade, a descrição de um determinado fenômeno ou uma população, podendo ainda estabelecer relações com variáveis $^{8}$.

Entre os meses de agosto de 2020 a fevereiro de 2021 foi realizado o cuidado em saúde focado na oferta de alimentos de preferência de duas usuárias de internação psiquiátrica em dois hospitais do Distrito Federal (DF), sendo um hospital geral e um psiquiátrico.

Embora o hospital psiquiátrico seja considerado como integrante da Rede de Atenção Psicossocial (RAPS), há incongruências na relação entre hospital psiquiátrico e melhora na 
saúde de indivíduos submetidos a internação involuntária e expostos a privação de liberdade e às regras institucionais ${ }^{9,6}$.

De acordo com a Portaria $N^{\circ} 3.088$ de 2011, fazem parte da RAPS, a Atenção Primária em Saúde, os Centros de Atenção Psicossocial, os serviços de atendimentos a urgência e emergência, os serviços de atenção residencial de caráter transitório e a atenção hospitalar na qual os hospitais gerais devem possuir leitos para pessoas com demandas psiquiátricas. Tais serviços colaboram para as desinstitucionalização e reintegração social das pessoas em sofrimento psíquico ${ }^{10}$.

Com a promulgação da Norma Técnica $N^{\circ} 11$ de 2019, a saúde mental do Brasil, ameaça retroceder décadas de conquistas, ao dar protagonismo à presença de hospitais psiquiátricos dentro da RAPS. Os hospitais psiquiátricos favorecem o estigma da loucura, a exclusão social e ainda favorecem as violências e violações dos Direitos Humanos ${ }^{5,9}$.

Durante o período, foram acompanhadas duas usuárias, sendo uma natural de Fortaleza-CE e outra do estado de Goiás, ambas com diagnóstico psiquiátrico de esquizofrenia, submetidas à internação involuntária, onde mesmo após estabilização do quadro e aptas à alta hospitalar permaneciam internadas. A prorrogação da alta se deu por meio de burocracias institucionais, que as impediam de saírem do ambiente hospitalar por falta de vínculo, suporte familiar além da fragilidade da RAPS no DF que ainda possui residências terapêuticas entre os dispositivos substitutivos ao hospital psiquiátrico, gerando, assim, aumento do sofrimento, e como consequência sentimentos recorrentes de tristeza, desânimo, e inapetência fortalecida por medidas de controle como contenção mecânica e medicamentosa frequentes e de longa duração.

Entre as usuárias, uma apresentava rebaixamento de humor frequente por sentimento de abandono, e a outra, inapetência devido à internação prolongada, com perda de peso 
ponderal de aproximadamente $10 \%$ do peso em dois meses de internação, com sinais visíveis de desnutrição energético-proteica e eletividade para início de nutrição enteral ${ }^{11}$.

A abordagem se deu através da escuta qualificada durante as vivências entre profissional de saúde e usuárias, buscando entender o contexto que o levou a tal situação, além de conhecer as preferências alimentares. Para Santos ${ }^{12}$, a escuta qualificada se faz um importante instrumento capaz de transformar de forma revolucionária o cuidado em saúde mental, possibilitando a humanização e o melhor tratamento e reabilitação além de proporcionar a integralidade do cuidado, considerando os aspectos biológicos e sociais que se relacionam com o indivíduo.

No decorrer do acompanhamento houve a facilidade da comunicação com as usuárias, e como obstáculo, havia a dificuldade de fomentar a esperança de liberdade, sendo uma delas recusada diversas vezes por instituições de longa permanência para idosos devido o diagnóstico psiquiátrico. No hospital psiquiátrico, a maior dificuldade encontrada durante o cuidado nutricional, foi resistência dos profissionais da assistência, que frequentemente usavam de práticas de contenção e desmoralizavam a oferta dos alimentos ofertados pela equipe de nutrição quando baseados na preferência da usuária.

Em uma das pacientes, foi identificado à preferência por farofa, sendo solicitada nas duas principais refeições, e no outro caso a preferência se dava pela preparação brasileira popularmente conhecida como coxinha, além de pão com ovo e pão com carne moída, sendo então solicitados às unidades de alimentação e nutrição dos hospitais, como guarnição no primeiro caso, e substituto ao almoço e jantar no outro, a fim de melhorar a aceitação da dieta, estabilização do peso e manutenção da via oral para alimentação.

\section{RESULTADOS E DISCUSSÃO}

A cada mordida, sabor de saudade 
Durante os diálogos entre residente e usuárias, ao ser abordado o porquê de determinada comida ser frequentemente solicitada, entre as justificativas dadas havia referência a lembranças da infância, em um contexto de perda materna e a consequente transição de cuidado então passado à irmã mais velha que assim como a mãe, realizava a preparação da comida. Tal situação fortaleceu o elo entre as irmãs, sendo a comida um paralelo entre perda e conforto.

Estar internado implica em se distanciar da rotina, de locais conhecidos, de pessoas amadas, representa uma situação de ruptura total de tudo aquilo que faz parte do indivíduo em seu sentido mais amplo de ser e existir. Mesmo após anos de exposição das violências das instituições totais, o hospital, sobretudo o hospital psiquiátrico, continua a fazer parte dos instrumentos considerados adequados no cuidado em saúde mental. Para Amarante, o hospital psiquiátrico representa a exclusão do indivíduo do meio familiar e de seu território, evidenciando a doença sobre o indivíduo, suprimindo sua identidade e singularidade por meio das relações de poder ${ }^{6}$.

Quando analisado simbolicamente, a cozinha é vista por muitas pessoas como o coração da casa. Nela há o fogo que aquece os alimentos a serem preparados, aquece o ambiente, e aquece os relacionamentos entre os que a frequentam ${ }^{13}$. As trocas ocorridas na cozinha, em nenhum momento se desvinculam da comida, mas trazem mais significados à comida e ao comer, e, possibilitam a construção das relações familiares por conectar gerações, não apenas num binômio mãe e filho, mas uma ligação ampliada com o passado, com pessoas anteriores à estas por meio do ensinamento de preparações culinárias e modos de cozinhar e comer $^{1,14}$.

Com o decorrer do crescimento e desenvolvimento, as relações durante as refeições se expandem, não sendo mais uma relação apenas entre mãe e filho, mas é adicionado a este 
momento outras figuras da configuração familiar, e outras pessoas do convívio. Apesar de nem sempre os momentos durante a alimentação serem harmônicos entre as relações interpessoais, a presença de conflitos faz parte de todas as relações sociais, entretanto momentos de felicidade e comemoração coletivas fortalecem a presença de afeto durante a alimentação ${ }^{3}$.

Segundo Pollan ${ }^{14}$ por meio da alimentação a criança aprende a compartilhar, a esperar sua vez, a falar e ouvir, entende como as relações familiares se desenvolvem. É ainda, em todo o processo da realização das refeições, com toda troca de afetos que as memórias se desenvolvem e se firmam, proporcionando novas formas de sentir o alimento, não apenas por meio da oralidade que envolve o comer, mas de forma intangível e ampla ${ }^{13}$.

De acordo com Barcelos ${ }^{13}$ o gosto é complexo e misterioso, sendo associado ao tato em nível profundo onde deixa-se de apenas sentir as texturas e demais sensações físicas dentro da boca, mas por meio dele o sabor das coisas chega à alma, podendo ser acessadas memórias não voluntárias, como recordações da memória da alma. Por meio dos sabores os processos de vida são objetivados à alma, tornado os sabores um elo de associação com o mundo.

\section{Isso é comida de gente como eu}

Outra justificativa apontada por uma das usuárias foi a ligação do alimento de sua preferência com sua raça/cor negra. O alimento em questão tratava-se de farinha de mandioca, um alimento comum na alimentação do brasileiro principalmente nas regiões norte e nordeste de acordo com a POF 2008-2009, e que nessa situação, foi considerado pela usuária como indispensável dentro da rotina alimentar, e apontado como algo inseparável de quem era e como garantidor de força e energia durante o dia ${ }^{15}$. 
Quando observado o contexto étnico-racial do Brasil, fica evidente que se trata de um país majoritariamente negro, onde a maior parte dos usuários do Sistema Único de Saúde SUS é de cor negra, algo que deve ser sempre levado em consideração pela equipe de saúde, sobretudo as singularidades dessa população. O racismo estrutural, principalmente, encarcera essas pessoas em situações de maior vulnerabilidade, tirando-lhes direitos e acessos como segurança, educação e boas condições de trabalho, e condicionando a população negra à marginalização e péssimas condições socioeconômicas, impactando diretamente na saúde e alimentação $^{16}$.

Entre as classes mais baixas, é presente a noção de que alimento bom é aquele que dá forças para o trabalho durante todo o dia, sendo estes alimentos comumente conhecidos como alimentos provedores de "sustança", como é o caso da farinha de mandioca. Além disso, outro fator importante associado ao consumo e preferências por esses alimentos é seu menor custo comparado com outros grupos alimentícios, sendo consumido por grande parte das famílias com menores condições socioeconômicas e acompanhados de alimentos base da alimentação do brasileiro como o arroz e feijão, sendo a cultura e o regionalismo aspectos também importantes na determinação de preferências alimentares ${ }^{17}$.

De acordo com Klotz-Silva, Prado e Seixas ${ }^{18}$ alimento quando consumido em repetição, dando a ideia de uma rotina, padronização ou hábito, não deve ser visto superficialmente. A alimentação individual e coletiva, são processos construídos ao longo do tempo, variando a depender da região geográfica, e de contextos sociais, culturais e históricos, de modo que quando uma preferência alimentar for considerada um hábito alimentar devido sua frequência, tais expressões e considerações devem ser questionadas a fim de buscar entender o indivíduo em seu sentido biopsicossocial e sua relação com esse alimento, evitando o reducionismo dessa relação ao campo biológico ou no sentido naturalizador de processos complexos e singulares. 


\section{“Mas eu sempre comi isso!”}

Comer é necessário fisiologicamente para a sobrevivência humana, entretanto, o comer adquire novas funções a partir das vivências humanas intrinsicamente associadas à sociabilidade, como a determinação de identidade. Com a comensalidade as relações pessoais com a comida começaram a se desenvolver, sendo as trocas de afetos no momento da refeição um elemento relevante, tais relações referenciam e ajudam a formular as preferências e gostos alimentares do indivíduo, possibilitando formas singulares e coletivas de alimentação que vão desde a preparação, ao consumo e até a forma de consumir, formulando-se assim comportamento cultural e fornecendo identidade alimentar ${ }^{1}$.

A diversidade alimentar no Brasil é vasta e marcada por inúmeras culturas, alimentos, preparações e modos de fazer a comida, do solo à mesa. Em 2014 com a publicação da atualização do Guia Alimentar para a População Brasileira - GAPB, não apenas uma nova forma de classificar os alimentos foi agregado à visão da nutrição, mas houve espaço para a autonomia da população em fazer suas próprias escolhas, podendo ser baseada na classificação dos alimentos, assim como na valorização do regionalismo e culturas alimentares consideradas como fomentadoras do sentimento de pertencimento dos indivíduos, e capazes de agregar bem-estar, devendo a ciência da nutrição ampliar a visão a fim de valorizar não apenas o aspecto biológico da alimentação, mas também os aspectos sociais, culturais e simbólicos da alimentação ${ }^{19,1,20}$.

Embora uma internação seja entendida como possibilidade de atenção e cuidados em saúde adequados, visando um contexto de melhora do estado de saúde, a pessoa submetida ao regime de internação ainda mantém seus direitos civis e humanos garantidos, podendo assim participar do seu próprio cuidado e decidir sobre seu tratamento ${ }^{21}$. 
A autonomia é um princípio bioético, que preconiza a capacidade da pessoa gerir seu próprio eu, podendo suas escolhas e decisões serem baseadas em interesses e vontades próprias, sendo assim, os profissionais de saúde devem respeito a existência humana dentro de sua complexidade, de modo a validar e apoiar com ações respeitosas a autonomia da pessoa sob seus cuidados, o que não significa estar isento de responsabilidades, mas prestar as devidas informações necessárias, de forma compreensível e com qualidade, de acordo com o estabelecido pelo usuário, sendo o respeito à autonomia uma obrigação moral dos profissionais de saúde ${ }^{21}$.

Os fatores que impactam na qualidade da alimentação de um indivíduo são diversos, sendo de caráter ambientais, políticos e sociais, desse modo o cuidado necessita ser transdisciplinar, para além da internação, e na perspectiva do cuidado em saúde mental pautado na reabilitação psicossocial, garantir a autonomia e fortalecer a cidadania são elementos importantes que podem impactar positivamente na saúde do indivíduo ${ }^{20,6}$.

Há tempos é sabido que a internação hospitalar prolongada se associa com maiores índices de desnutrição, com consequente piora no prognóstico, maiores riscos a infecções, maiores taxas de óbitos e aumento dos custos hospitalares. Frente a isso, a desnutrição é um desafio a ser superado e evitado no contexto hospitalar, sendo a triagem nutricional na admissão e reavaliações periódicas, formas necessárias para prevenir e proporcionar a intervenção nutricional precoce, assim como estratégias que garantam a boa aceitação alimentar durante a internação ${ }^{11,22}$.

As estratégias para a melhor aceitação alimentar devem ser reinventadas e reavaliadas, de forma a se adaptar ao usuário, tendo em vista que a internação é um evento estressor, e o distanciamento da rotina alimentar pode se apresentar com a redução da aceitação, ou mesmo rejeição da dieta hospitalar por diversos motivos, incluindo questões psicológicas. A 
Gastronomia hospitalar é uma vertente que vem ganhando espaço e somando forças contra a desnutrição, por considerar a comida com seus valores além do biológico e valorizar os aspectos sensoriais das preparações, possibilitando unir as preferências alimentares com uma dieta saudável por meio de adaptações que podem ser realizados na cozinha hospitalar ${ }^{22}$.

\section{CONSIDERAÇÕES FINAIS}

Comer é mais que fornecer nutrientes ao corpo, é um reforço de identidade cultural, um resgate de memórias e afetos com o mundo e seus laços sociais. Dessa forma, o cuidado nutricional deve se amparar não apenas nos aspectos biológicos, mas também em aspectos culturais, sociais, psicológicos e antropológicos da alimentação, de modo a proporcionar um cuidado ampliado que abarque os múltiplos fatores que podem influenciar no estado de saúde.

Um cuidado terapêutico nutricional, não deve se basear apenas em protocolos de condutas, ele deve ser flexível e buscar se adequar ao indivíduo dentro de sua subjetividade, mas para que isso ocorra é necessário romper com o modelo biomédico tradicional, que estabelece relações verticais entre usuário e profissional de saúde. A autonomia do usuário em escolher a melhor forma de tratamento não é apenas um dever, mas indispensável por proporcionar a cogestão e o autocuidado, além de ferramenta que pode ser associada à reabilitação psicossocial, garantia de cidadania e respeito por proporcionar as relações horizontalizadas no cuidado em saúde.

Diante da singularidade do indivíduo, proporcionar as escolhas alimentares ao paciente respeitando suas preferências, possibilita a garantia da sua manifestação de identidade, cultura e manutenção de sua rotina alimentar, favorecendo o bem-estar, manutenção da alimentação e contato afetivo com seu território. 
Os afetos relacionados à alimentação, devem ser considerados pelo nutricionista, por ser um aspecto importante na definição de preferências alimentares, e que podem ser explorados de forma a auxiliar nas condutas com vista a manutenção, prevenção e recuperação do estado de saúde, por meio de informações adequadas e adaptações das preparações, sem a necessidade de excluir as comidas da rotina do usuário. Diante do exposto, mas estudos sobre a temática se fazem necessários para o melhor entendimento de como e em quais dimensões a alimentação afetiva pode influenciar o estado de saúde.

\section{REFERÊNCIAS}

1. Lima RS, Neto JA, Farias RC. Alimentação, comida e cultura: o exercício da comensalidade. Demetra: Alimentação, Nutrição \& Saúde. 2015;10(3):507-22. Available from: https://doi.org/10.12957/demetra.2015.16072

2. Moreira SA. Alimentação e comensalidade: aspectos históricos e antropológicos. Ciência e $\begin{array}{llll}\text { Cultura. } & \text { 2010;62(4):23-26. Available from: }\end{array}$ http://cienciaecultura.bvs.br/scielo.php?script=sci_arttext\&pid=S0009$67252010000400009 \& \operatorname{lng}=\mathrm{en}$.

3. Romanelli G. O significado da alimentação na família: uma visão antropológica. Medicina (Ribeirão Preto). 2006;39(3):333-39. Available from: https://www.revistas.usp.br/rmrp/article/view/388

4. Pais SC, Ferreira PD (Orgs). A redução das assimetrias sociais no acesso à alimentação e à saúde: Políticas e práticas. Porto: Mais Leitura; 2016. 108 p.

5. Almeida JM. Política de saúde mental no Brasil: o que está em jogo nas mudanças em curso. Cad. Saúde Pública. 2019;35(11). Available from: https://doi.org/10.1590/0102311X00129519 
6. Amarante P. Saúde mental e atenção psicossocial. 4. Ed. Rio de Janeiro: Editora Fiocruz; 2007.123p.

7. Minayo MC (Org.). Pesquisa social: teoria, método e criatividade. Petrópolis: Vozes, 2001.

8. Gil AC. Métodos e técnicas de pesquisa social. 6.ed. São Paulo: Atlas; 2008.

9. Brasil. Ministério da Saúde. Secretaria de Atenção à Saúde. Nota Técnica no 11/2019. Esclarecimentos sobre as mudanças na Política Nacional de Saúde Mental e nas Diretrizes da Política Nacional sobre Drogas. Brasília, 2019. Available from: https://pbpd.org.br/wp-content/uploads/2019/02/0656ad6e.pdf 10. Brasil. Ministério da Saúde. Portaria No 3.088, de 23 de dezembro de 2011. Institui a Rede de Atenção Psicossocial para pessoas com sofrimento ou transtorno mental e com necessidades decorrentes do uso de crack, álcool e outras drogas, no âmbito do Sistema Único de Saúde (SUS). Brasília, 2011. Available from: https://bvsms.saude.gov.br/bvs/saudelegis/gm/2011/prt3088_23_12_2011_rep.html 11. Toledo DO et al. Campanha "Diga não à desnutrição": 11 passos importantes para combater a desnutrição hospitalar. CEP. 2018;33(1):86-100. Available from: http://arquivos.braspen.org/journal/jan-fev-mar-2018/15-Campanha-diga-naoaadesnutricao.pdf

12. Santos AB. Escuta qualificada como ferramenta de humanização do cuidado em saúde mental na Atenção Básica. APS em Revista. 2019;1(2):170-79. Available from: https://doi.org/10.14295/aps.v1i2.23

13. Barcellos Gustavo. O banquete de psique: Imaginação, cultura e psicologia da alimentação. Petrópolis: Editora Vozes; 2017. 180p.

14. Pollan, M. Cozinhar: uma história natural da transformação. 1. Ed. Rio de Janeiro: Editora Intrínseca; 2014. 554p 
15. Instituto Brasileiro de Geografia e Estatística. Pesquisa de Orçamentos Familiares 2008$2009-$ POF. Rio de Janeiro, 2010. Available from: https://biblioteca.ibge.gov.br/visualizacao/livros/liv50063.pdf

16. Barbosa RR, Silva CS, Sousa AA. Vozes que ecoam: racismo, violência e saúde da população negra. Revista Katálysis. 2021;24:353-63. Available from: https://doi.org/10.1590/1982-0259.2021.e77967

17. Bertoncelo E. Classe social e alimentação: padrões de consumo alimentar no Brasil contemporâneo. Revista Brasileira de Ciências Sociais. 2019;34(100). Available from: https://doi.org/10.1590/3410005/2019

18. Klotz-Silva J, Prado SD, Seixas CM. A força do" hábito alimentar": referências conceituais para o campo da Alimentação e Nutrição. Physis: Revista de Saúde Coletiva. 2017;27:1065-85. Available from: https://doi.org/10.1590/S0103-73312017000400011

19. Oliveira MS, Santos LA. Guias alimentares para a população brasileira: uma análise a partir das dimensões culturais e sociais da alimentação. Ciência \& Saúde Coletiva. 2020;25:2519-28. Available from: https://doi.org/10.1590/1413-81232020257.22322018

20. Brasil. Ministério da Saúde. Secretaria de Atenção à Saúde. Departamento de Atenção Básica. Guia alimentar para a população brasileira. Departamento de Atenção Básica. 2. ed. $\quad$ Brasília, 2014. Available from: https://bvsms.saude.gov.br/bvs/publicacoes/guia_alimentar_populacao_brasileira_2ed.pdf 21. Rego Sergio, Palácios M, Siqueira-Batista R. Bioética para profissionais da saúde 1. Ed. Editora Fiocruz, 2009. 160 p.

22. Souza MD, Nakasato M. A gastronomia hospitalar auxiliando na redução dos índices de desnutrição entre pacientes hospitalizados. O mundo da saúde. 2011;35(2):208-14. Available from: 
https://bvsms.saude.gov.br/bvs/artigos/gastronomia_hospitalar_auxiliando_reducao_desnutric ao_pacientes.pdf 